\title{
Fully Analog ECG Baseline Wander Tracking and Removal Circuitry using HPF Based R-peak Detection and Quadratic Interpolation
}

\author{
Masoud Nazari $^{1^{*}}$, Alireza Bagheri Rajeoni ${ }^{1}$, Kye-Shin Lee ${ }^{1}$
}

\begin{abstract}
This work presents a fully analog baseline wander tracking and removal circuitry using high-pass filter (HPF) based R-peak detection and quadratic interpolation that does not require digital post processing, thus suitable for compact and low power long-term ECG monitoring devices. The proposed method can effectively track and remove baseline wander in ECG waveforms corrupted by various motion artifacts, whereas minimizing the loss of essential features including the QRS-Complex. The key component for tracking the baseline wander is down sampling the moving average of the corrupted ECG waveform followed by quadratic interpolation, where the R-peak samples that distort the baseline tracking are excluded from the moving average by using a HPF based approach. The proposed circuit is designed using CMOS 0.18 $\mu \mathrm{m}$ technology ( $1.8 \mathrm{~V}$ supply) with power consumption of $19.1 \mu \mathrm{W}$ and estimated area of $15.5 \mathrm{~mm}^{2}$ using a $4^{\text {th }}$ order HPF and quadratic interpolation. Results show SNR improvement of $10 \mathrm{~dB}$ after removing the baseline wander from the corrupted ECG waveform.
\end{abstract}

Key Words: ECG baseline wander tracking and removal, High-pass filter, Moving average, Quadratic interpolation.

\section{INTRODUCTION}

The conventional stationary Electrocardiography (ECG) monitoring can reveal harmful cardiac abnormalities; however, it is not feasible for long term non-stationary recording for detecting transient loss of consciousness (TLOC), lightheadedness or palpitations which occur only once or twice a month or even a few times per year [1]. As a result, for long term and continuous real-time ECG recording, there is an increasing demand for ambulatory ECG monitoring that can effectively record ECG signals while the patient is performing routine activities such as working, exercising or sleeping without interfering with their activities. However, as shown in Fig. 1, the quality of continuous long term ECG monitoring is critically degraded by baseline wander originating from motion artifacts due to various body movements that alters the impedance between the skin and the sensing electrode at the input of the ECG sensor frontend. Baseline wander leads to difficult interpretation of the ECG waveforms, since the basic features (like QRS-Complexes) will not be clearly detectable. As such, tracking and removing the baseline wander is required for proper operation of the continuous real-time ECG monitoring device as it may cause false alarms or wrong event detections that trigger emergency

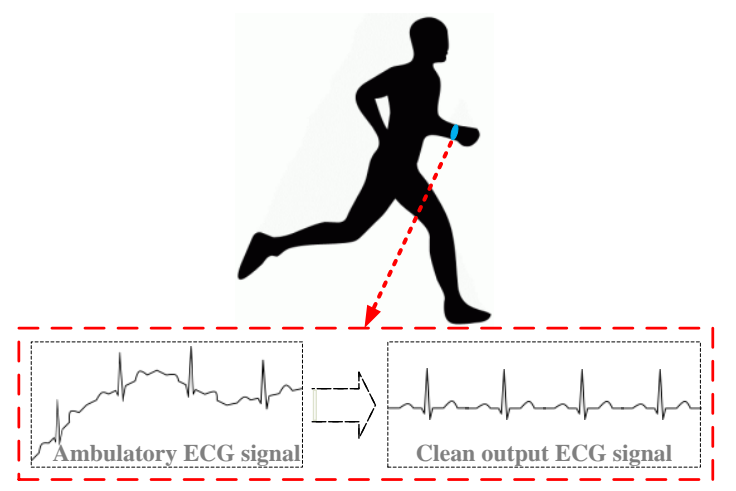

Fig. 1. Ambulatory ECG monitoring with ECG waveform corrupted by motion artifact and clean ECG waveform obtained by baseline wander tracking and removal.

treatments.

Numerous software and hardware based ECG baseline wander elimination schemes have been presented in the literature including the empirical decomposition method [2], block least mean square (BLMS) algorithm that use motion acceleration as the reference signal [3], and Hilbert vibration decomposition (HVD) [4]. However, the above methods rely on post processing of the corrupted ECG waveforms, thus are not suitable for continuous real-time monitoring. Meanwhile, adaptive filter [5] and independent

Manuscript received August 14, 2020; Revised September 13, 2020; Accepted September 17, 2020. (ID No. JMIS- 20M-08-021) Corresponding Author (*): Masoud Nazari, ASEC 260, The University of Akron, Akron, OH 44325, USA, 330-972-2996, mn78@zips.uakron.edu.

${ }^{1}$ Dept. of Electrical \& Computer Engineering, The University of Akron, Akron, Ohio, USA. 
component analysis (ICA) [6] based hardware schemes are available, but these require additional components such as micro-controllers, FPGAs, ADCs, and DACs which increases the power consumption and form factor of the ECG monitoring device.

Nowadays, Brain-Computer Interfaces (BCI) which use artificial intelligence (AI) algorithms such as deep learning [7] are widely utilized in recording ECG/EEG signals and recognizing their features. However, a hybrid classifier model used in [8] and a pattern-based authentication method utilized in [9] need long short-term memory along with recorded EEG/ECG waveforms to classify the upcoming signals for noise reduction. These approaches impose latency between different hardware modules, high computation, more area and power consumption to the whole systems. In this paper, a fully analog baseline wander tracking and removal circuitry is proposed. The proposed circuitry that can be incorporated within the ECG sensor frontend without using digital post processing, is suitable for continuous real-time ECG monitoring devices. In addition, the baseline wander in ECG waveforms corrupted by various motion artifacts can be effectively tracked and removed, whereas minimizing the loss of essential features including the QRS-Complex. This paper is organized as follows. Section 0 describes the proposed ECG baseline wander tracking and removal scheme. The circuit implementation and simulation results are shown in section III and IV, followed by the conclusions in section V.

\section{ECG BASELINE WANDER TRACKING AND REMOVAL}

The proposed ECG baseline wander tracking and removal scheme can be easily incorporated in the sensor frontend. Fig. 2 shows the block diagram of the proposed circuitry which includes two delays, baseline detection block, and the subtractor. Once the corrupted ECG signal is amplified by $\mathrm{A}_{1}$, it will go through the baseline detector that extracts the baseline wander which is subtracted from the delayed version of the corrupted signal to generate a clean waveform. The final output is then amplified by another gain stage $A_{2}$ for further processing. The basic concept of baseline wander detection is taking the moving average of the corrupted ECG signal. The baseline detector consists of the high pass filter (HPF), moving average and interpolation blocks. The HPF passes high frequency (HF) components of ECG signal in particular, the R-peaks, and

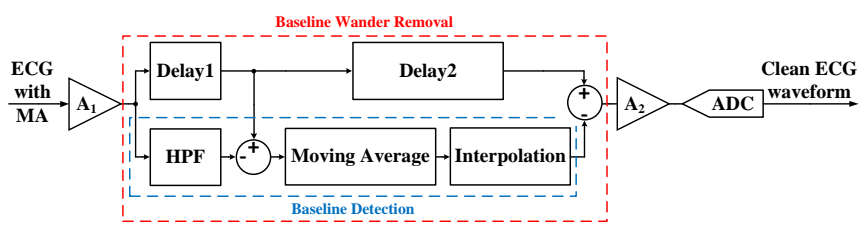

Fig. 2. The proposed baseline wander elimination scheme.

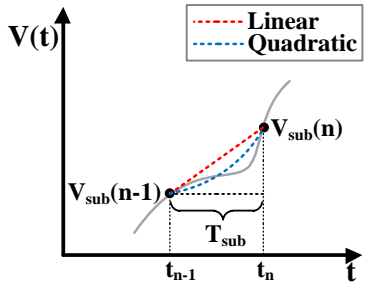

Fig. 3. Linear and quadratic interpolations with two data points.

attenuates low frequency (LF) components - motion artifacts and DC-offset. However, the capability of the HPF for attenuating LF components and passing HF components such as the R-peaks will depend on the filter order. Therefore, subtracting the HPF output from the corrupted ECG waveform will exclude R-peak samples from the moving average, where Delay 1 is used for compensating the HPF delay. This will prevent distortion in the baseline wander tracking caused by the R-peaks, once they are included in the moving average. Finally, the baseline wander is extracted by linearly or quadratically interpolating the HF components excluded down sampled moving average, and subtracted from the delayed version (through Delay2) of the corrupted ECG waveform to generate a clean ECG signal.

Fig. 3 shows the concept of linear and quadratic interpolation methods used in the proposed approach to track the baseline wander in corrupted ECG signals. The baseline level of the incoming ECG signal from moving average is obtained by continuous linear or quadratic interpolations of the two data points $\mathrm{V}_{\text {sub }}(\mathrm{n}-1)$ and $\mathrm{V}_{\text {sub }}(\mathrm{n})$

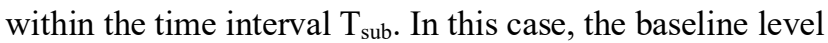
$\mathrm{V}_{\text {Lerp }}, \mathrm{V}_{\text {Linear }}$ and the quadratic interpolation output $\mathrm{V}_{\text {Quad }}$ between time $t_{n-1}$ and $t_{n}$ can be expressed as:

$$
\begin{aligned}
V_{\text {Lerp }}= & \frac{1}{T_{\text {sub }}} \cdot \int_{t_{n-1}}^{t}\left[V_{\text {sub }}(n)-V_{\text {sub }}(n-1)\right] d t \\
& V_{\text {Linear }}=V_{\text {Lerp }}+V_{\text {sub }}(n-1) \\
V_{\text {Quad }}= & \frac{2}{T_{\text {sub }}} \cdot \int_{t_{n-1}}^{t} V_{\text {Lerp }} d t+V_{\text {sub }}(n-1) .
\end{aligned}
$$

This will lead to linear and quadratic curves that connect the two points $\mathrm{V}_{\text {sub }}(\mathrm{n}-1)$ and $\mathrm{V}_{\text {sub }}(\mathrm{n})$. Therefore, the final equations for linear and quadratic interpolations are as follows:

$$
\begin{aligned}
& V_{\text {Linear }}=\frac{V_{\text {sub }}(n)-V_{\text {sub }}(n-1)}{T_{\text {sub }}} \cdot\left(t-t_{n-1}\right) \\
& +V_{s u b}(n-1) \\
& V_{\text {Quad }}=\frac{V_{\text {sub }}(n)-V_{\text {sub }}(n-1)}{T_{\text {sub }}^{2}} \cdot\left(t-t_{n-1}\right)^{2} \\
& +V_{\text {sub }}(n-1)
\end{aligned}
$$

Fig. 4 shows how the baseline wander is extracted from the corrupted ECG signal to obtain a clean waveform [10]. 


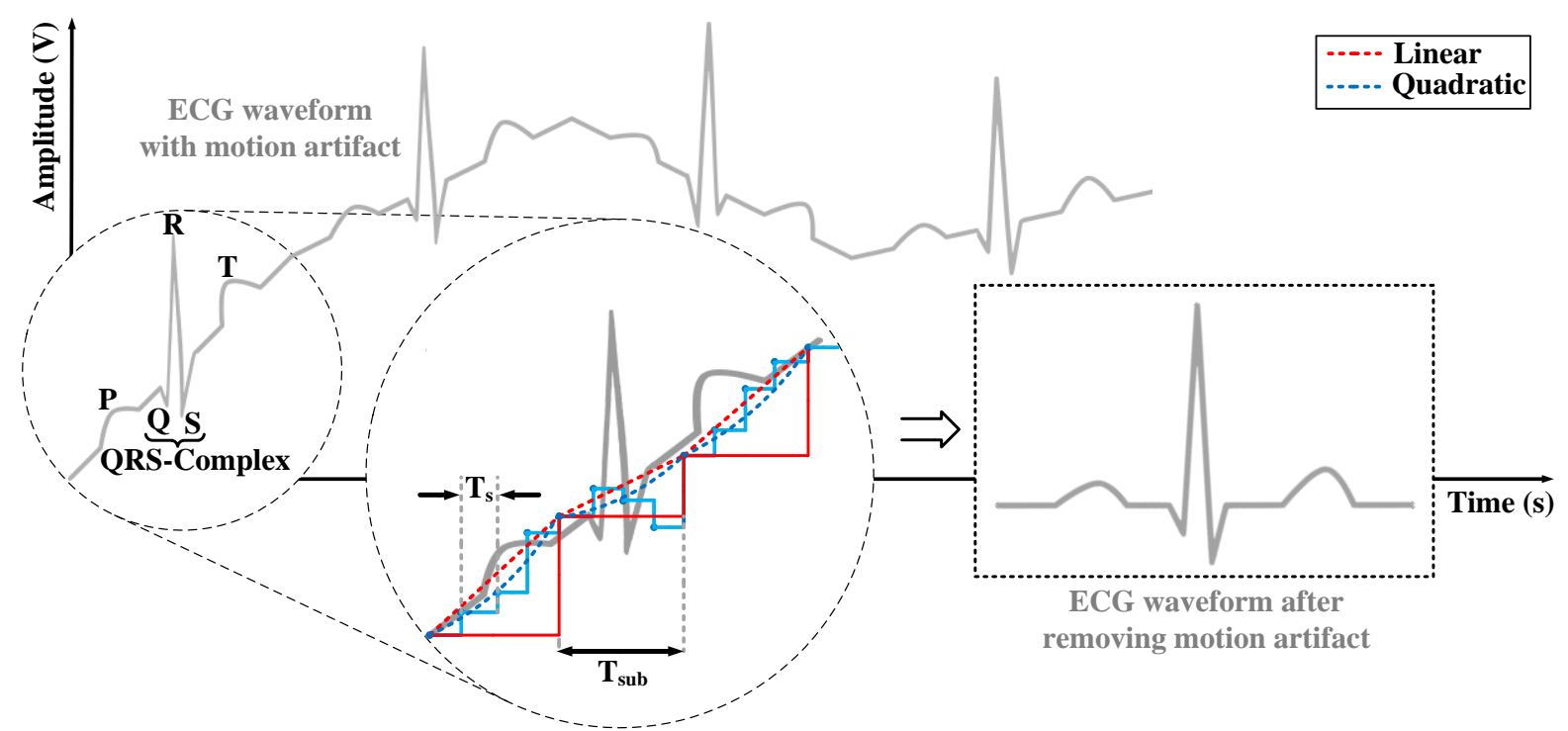

Fig. 4. Baseline wander extraction using moving average, linear or quadratic interpolations.

The moving average excluding the R-peaks and HF components sampled with $\mathrm{f}_{\mathrm{s}}\left(=1 / \mathrm{T}_{\mathrm{s}}\right)$ (the blue solid line waveform) is down sampled with $\mathrm{f}_{\text {sub }}\left(=1 / \mathrm{T}_{\text {sub }}\right)$, which results in the red solid waveform where generally, $f_{s}>f_{\text {sub. }}$. The down sampled moving average is interpolated with linear or quadratic methods, where the red and the blue dotted waveforms shows the result of linear and quadratic interpolation, respectively. Since quadratic interpolation will better represent the baseline wander, quadratically interpolating the down sampled moving average will lead to improved baseline wander tracking compared to linearly interpolating the data points. The final clean output waveform will be obtained by subtracting the detected interpolated data points from the corrupted ECG waveform.

\section{CIRCUIT IMPLEMENTATION}

The proposed baseline wander tracking and removal circuitry which includes the HPF based R-peak detection and quadratic interpolation is designed using CMOS 0.18$\mu \mathrm{m}$ technology with supply voltage of $1.8 \mathrm{~V}$.

\subsection{High Pass Filter}

The HPF should effectively pass QRS-Complex of ECG signal and suppress LF components, thus the filter cut-off

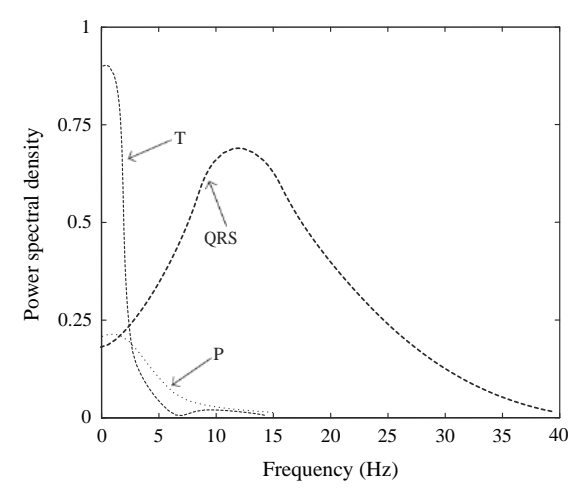

Fig. 5. Power spectra of high frequency components of ECG signal [11]. frequency should be set based on the frequency spectrum of the ECG signal. According to Fig. 5 presented in [11], QRSComplex can be well preserved by setting the cut-off frequency of HPF around $10-15 \mathrm{~Hz}$, where the proposed filter is designed based on this assumption. However, it should be noted that increasing the order of HPF will further attenuate LF components of ECG signal which results in better R-peaks detection. Therefore, with a higher order filter, R-peaks are better excluded from moving average by subtracting the HPF output from the delayed version of corrupted ECG signal. In addition, the P-and T-waves will be excluded from the baseline wander through down sampling and interpolating the moving average. The $\mathrm{z}-$ domain transfer function of the 4-th order HPF is given as:

$$
G(z)=\frac{Y}{X}=\frac{a z^{4}+b z^{3}+c z^{2}+d z+e}{z^{4}} .
$$

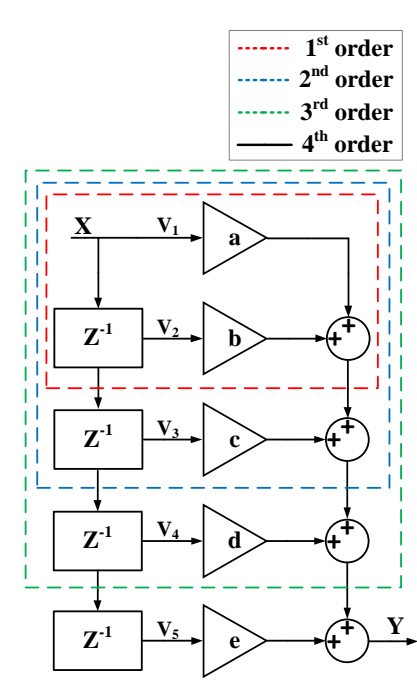

(a)

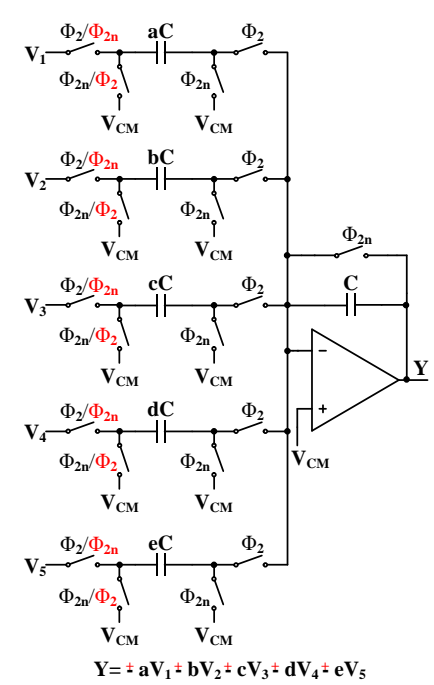

(b)
Fig. 6. (a) z-domain representation of the HPF (b) switch capacitor implementation of the $4^{\text {th }}$ order HPF. 
where $\mathrm{a}, \mathrm{b}, \mathrm{c}, \mathrm{d}$ and e coefficients are set to achieve 10-15 $\mathrm{Hz}$ cut-off frequency and attenuate LF components while keeping the filter stable. Fig. 6(a) shows the z-domain representation of the HPF which includes delays $\left(\mathrm{z}^{-1}\right)$, gain stages, and adders, and Fig. 6(b) shows the switch capacitor (SC) implementation of the $4^{\text {th }}$ order HPF, where the four adders are combined into one single adder with five inputs. Furthermore, the gain stages (a to e) are realized by adjusting the ratio between each input capacitor and the feedback capacitor (C). However, since the coefficients a to e can be either negative or positive, their signs are inverted by swapping the control clock of the first two switches. As a result, the positive and negative coefficients can be implemented by setting the adder to a non-inverting or inverting SC summing configuration.

\subsection{Delay and Subtractor}

Fig. 7(a) shows the track and hold $(\mathrm{T} / \mathrm{H})$ or delay $\left(\mathrm{z}^{-1}\right)$ circuit that operates in two modes. In the track mode $\left(\Phi_{2}=\right.$ $\mathrm{H}$ and $\left.\Phi_{2 \mathrm{n}}=\mathrm{L}\right)$, the input is sampled at $\mathrm{C}_{1}$ and the output tracks the input $\left(\mathrm{V}_{\mathrm{I}}=\mathrm{V}_{\mathrm{O}}\right)$. In the hold mode $\left(\Phi_{2}=\mathrm{L}\right.$ and $\Phi_{2 \mathrm{n}}$ $=\mathrm{H})$, the input is disconnected and the output shows the last value sampled in $C_{1}$. Fig. 7(b) shows the subtractor which is utilized to subtract the HPF output from the delayed version of corrupted ECG signal. The signal $V_{2}$ is subtracted from $V_{1}$ in phase $\Phi_{2}$ and the result will be kept and sent to the output during phase $\Phi_{2 n}$. The OTA used in the ECG baseline detection block is a rail-to-rail folded cascode configuration with DC gain of $61 \mathrm{~dB}$ and GBW of 12.9 $\mathrm{MHz}$, and power consumption of $290 \mathrm{nW}$.

\subsection{Moving Average}

The moving average circuit is shown in Fig. 8 in which it is comprised of an adder, a gain stage and delay. The adder and gain block are combined together same as the circuit shown in Fig. 6(b) but with two inputs where the input

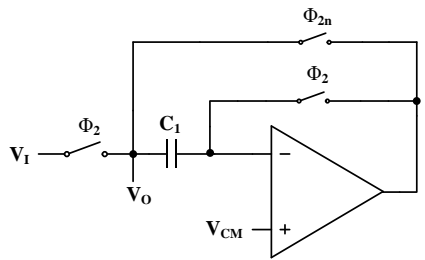

(a)

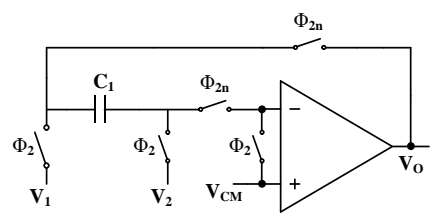

(b)
Fig. 7. (a) The $T / H /$ delay and (b) subtractor circuits.

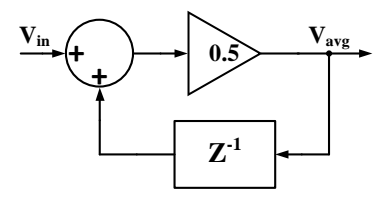

Fig. 8. The moving average circuit.

capacitors are half of the feedback capacitor (C) to satisfy 0.5 gain value. The delay block is also similar to Fig. 7(a).

\subsection{Quadratic Interpolation}

The quadratic interpolation block shown in Fig. 9(a) is realized using linear interpolation, subtractor, delay, offset cancellation, integrator and gain stage circuits. In the ideal behavioral model, the delay $\left(\mathrm{z}^{-1}\right)$ and offset cancellation circuits are not required. However, in the actual circuit, the delay block is used to compensate the delay generated by the subtractor in linear interpolation circuit and synchronize the signals $\mathrm{V}_{\text {Linear }}$ and $\mathrm{V}_{\text {sub }}(\mathrm{n}-1)$. In addition, the offset cancellation circuit removes the offset produced by the integrator coming from linear interpolation circuit. Overall, the blue dashed block in Fig. 9(a) implements eq. 1(c), which leads to the blue dotted waveform shown in Fig. 4. In this design, first, the delayed input $\left(\mathrm{V}_{\text {sub }}(\mathrm{n}-1)\right)$ is subtracted from the linearly interpolated data $\left(\mathrm{V}_{\text {Linear }}\right)$, which leads to $V_{\text {Lerp }}$ as shown in eq. 1(b). Next, after removing the offset from $\mathrm{V}_{\text {Lerp }}$, integrating with a gain of $2 / \mathrm{T}_{\text {sub }}$, and adding the presented initial value $\left(\mathrm{V}_{\text {sub }}(\mathrm{n}-1)\right)$, the $\mathrm{V}_{\text {Quad }}$ signal corresponding to eq. 1(c) or eq. 2(b) is generated.

The linear interpolation circuit is presented in [10]. The subtractor and delay circuits of quadratic interpolation block are same as Fig. 7. The integrator with the gain stage is implemented with a continuous time integrator as shown in Fig. 9(b), which consists of the pseudo resistor, capacitor and OTA. The pseudo resistor is in order of tens of Gigaohm to enforce the capacitor becomes on-chip and satisfy the gain value of $2 / \mathrm{T}_{\text {sub }}$. Next, the offset cancellation circuit is indicated in Fig. 9(c) where it contains a T/H and a subtractor. The $\mathrm{T} / \mathrm{H}$ circuit works with clock $\Phi_{3}$ with frequency of $\mathrm{f}_{\text {sub }}$ and $1 \%$ duty cycle. Therefore, initial data point of $V_{I}$ (in Fig. 9(c)) is sampled, kept and reduced from the upcoming $\mathrm{V}_{\mathrm{I}}$ signal for every $\mathrm{T}_{\text {sub }}$ cycle that generates $\mathrm{V}_{\mathrm{O}}$ without offset. Lastly, the combination of

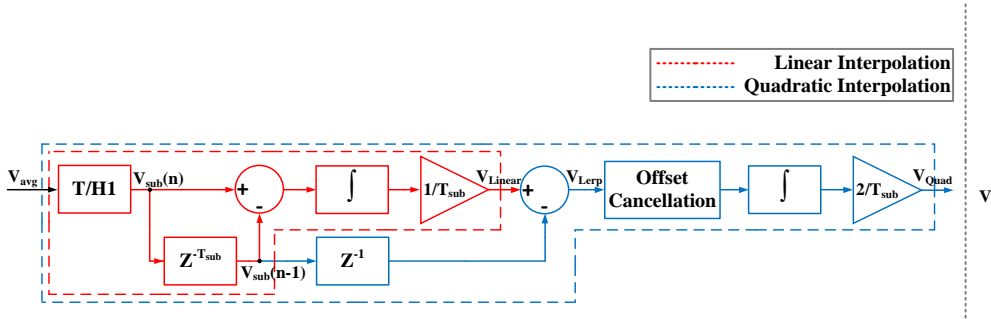

(a)

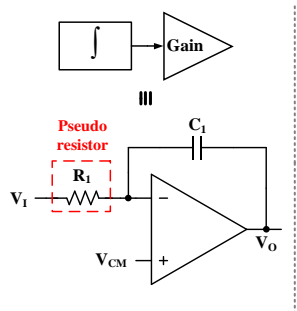

(b)
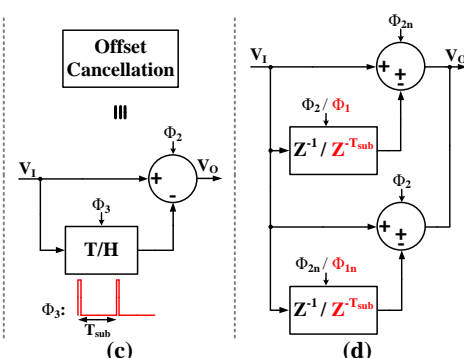

Fig. 9. Linear (red dashed line) and quadratic (blue dashed line) interpolation circuits, (b) the continuous time integrator with its intrinsic gain, (c) Offset cancellation circuit with timing of $\Phi 3$ clock signal, (d) pipeline operation of delay and Adder/subtractor circuits. 


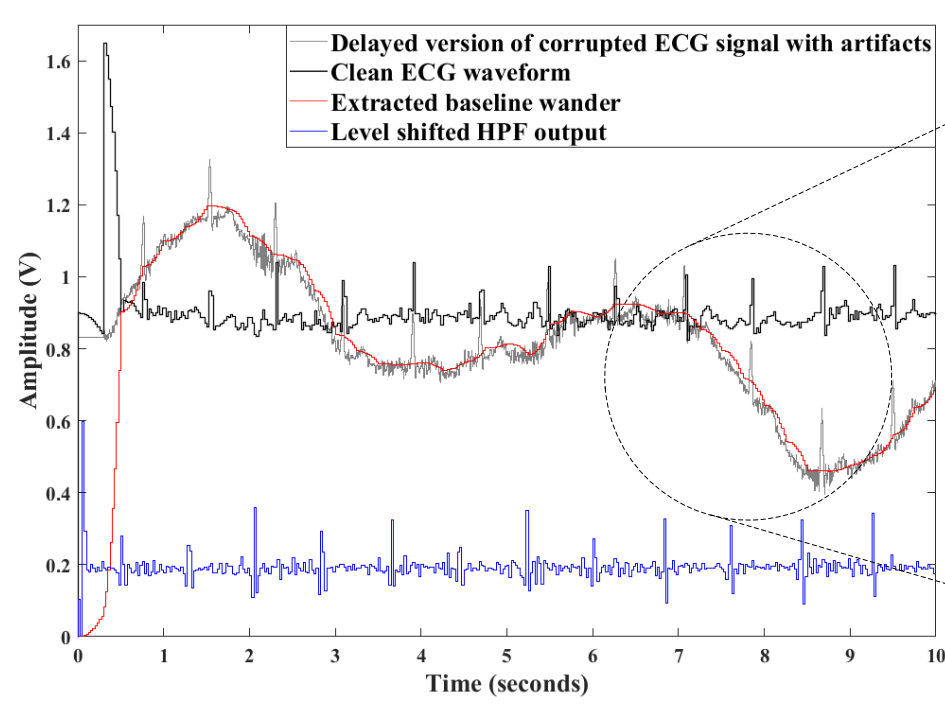

(a)

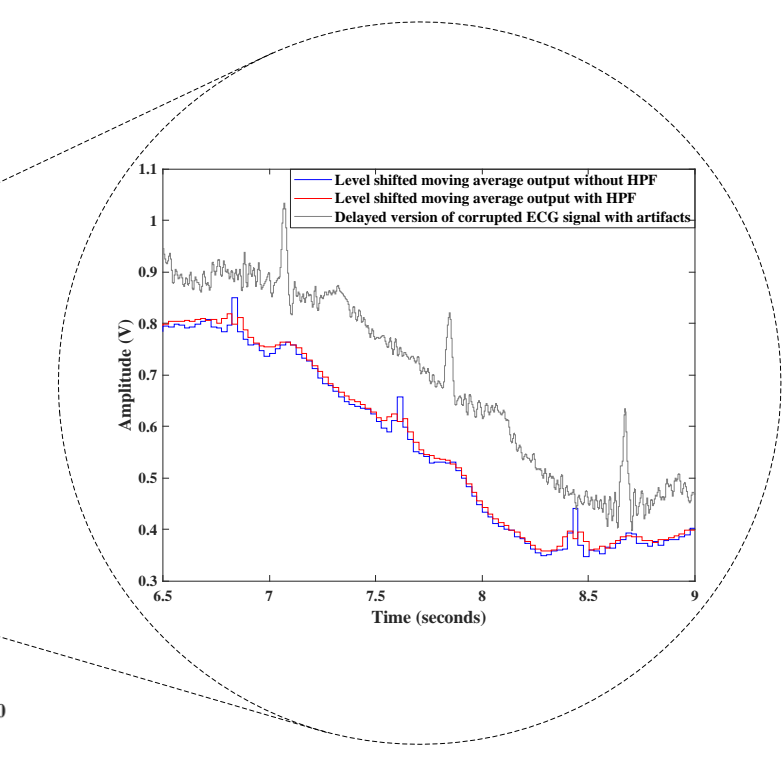

(b)

Fig. 10. (a) Corrupted ECG waveform with delay, 4th order HPF output with shifted level, extracted baseline level with quadratic interpolation and the final clean signal (b) the level shifted moving average output with and without using HPF.

adder/subtractor and delay circuits in the whole system operate in pipeline fashion as shown in Fig. 9(d). In this way, the signal can be tracked and followed for the whole clock cycle. This combination in the linear interpolation circuit uses $\Phi_{1}$ and $\Phi_{2}$ which are set to $\mathrm{f}_{\text {sub }} / 2$ and $\mathrm{f}_{\mathrm{s}} / 2$, respectively with duty cycle of $50 \%$, where $\Phi_{1 n}$ and $\Phi_{2 n}$ are the inverted version of $\Phi_{1}$ and $\Phi_{2}$. However, the delay $\left(\mathrm{z}^{-1}\right)$ and the $\mathrm{T} / \mathrm{H}$ circuit in the other parts operates with $\Phi_{2}$ clock signal, identical to the adder/subtractor.

\section{SIMULATION RESULTS}

The proposed ECG baseline wander tracking and removal circuitry is verified through circuit level simulation. The optimum value of $\mathrm{f}_{\text {sub }}$ is set to $4 \mathrm{~Hz}$, however, increasing $\mathrm{f}_{\text {sub }}$ will remove more useful components of ECG signal such as the QRS-Complex from the clean ECG output waveform. On the other hand, if $\mathrm{f}_{\text {sub }}$ is set lower than $4 \mathrm{~Hz}$, the baseline wander cannot be accurately tracked. Furthermore, the optimum value of $f_{s}$ is set to $40 \mathrm{~Hz}$, therefore the sampling rate of $\Phi_{1}$ and $\Phi_{2}$ clock results in $2 \mathrm{~Hz}$ and $20 \mathrm{~Hz}$ with $50 \%$ duty cycle. The sampling rate of $\Phi_{3}$ clock is set to $4 \mathrm{~Hz}$ with $1 \%$ duty cycle. The a-e coefficients of the $4^{\text {th }}$ order HPF are set to $-0.08,-0.26,0.67,-0.26$ and -0.08 respectively. Fig. 10(a) shows the waveforms obtained from the proposed ECG baseline wander tracking and removal circuitry with the $4^{\text {th }}$ order HPF and quadratic interpolation circuit. The gray waveform is the corrupted ECG signal (with delay), the blue waveform is the HPF output (with level shifted for clear view), red waveform is the extracted baseline wander, and the black waveform is the clean ECG signal generated from the proposed circuit. As shown, the final output waveform does not contain baseline wander and DC offset, whereas the key features of the ECG signals are well preserved. It should be noted that by increasing the order of HPF, the R-peaks are extracted more accurately while LF components are better attenuated. However, the odd-order HPFs which intrinsically contain odd number of adders/subtractors and delay elements, produce huge negative glitches when the R-peaks pass through the filter, which degrades the SNR improvement. Meanwhile, evenorder HPFs well preserve the R-peaks without producing much negative glitches. Fig. 10(b) shows the detected baseline wander (with level shifted for better view) after moving average stage with (the red waveform) and without (the blue waveform) using HPF to exclude R-peaks. Without the HPF, the moving average will not represent the true motion artifact due to the huge R-peak samples included in the moving average input, thus will distort the clean ECG waveform once the output of the interpolated down sampled moving average is subtracted from the corrupted ECG signal.

Table 1. Performance comparison of different filters and interpolation methods utilized in the proposed ECG baseline removal circuitry.

\begin{tabular}{|c|c|c|c|}
\hline $\begin{array}{c}\text { Filter } \\
\text { +Interpolation }\end{array}$ & Power $(\mu \mathrm{W})$ & $\begin{array}{c}\text { Core Area }^{*} \\
\left(\mathrm{~mm}^{2}\right)\end{array}$ & $\begin{array}{c}\text { SNR } \\
\text { improvement }(\mathrm{dB})\end{array}$ \\
\hline $\begin{array}{c}1^{\text {st }} \text { order HPF } \\
+ \text { Quadratic }\end{array}$ & 16.82 & $3.04 \times 4.12$ & 8.96 \\
\hline $\begin{array}{c}2^{\text {nd }} \text { order HPF } \\
+ \text { Quadratic }\end{array}$ & 17.4 & $3.17 \times 4.29$ & 9.21 \\
\hline $\begin{array}{c}3^{\text {rd }} \text { order HPF } \\
+ \text { Quadratic }\end{array}$ & 18.56 & $3.32 \times 4.49$ & 9.15 \\
\hline $\begin{array}{c}4^{\text {th }} \text { order HPF } \\
+ \text { Quadratic }\end{array}$ & 19.14 & $3.38 \times 4.58$ & $\mathbf{9 . 9 6}$ \\
\hline $\begin{array}{c}4^{\text {th }} \text { order HPF } \\
+ \text { Linear }\end{array}$ & 15.66 & $2.76 \times 3.75$ & 9.54 \\
\hline $\begin{array}{c}4^{\text {th }} \text { order LPF } \\
+ \text { No Interpol. }\end{array}$ & $\mathbf{6 . 3 8}$ & $\mathbf{1 . 1 7} \times \mathbf{1 . 5 9}$ & 7.58 \\
\hline
\end{tabular}

*Estimated chip area 
Table 1 shows the performance comparison results for distinct filter types/orders and interpolation methods. It is obvious that power consumption and estimated area will increase in accordance with elevating the HPF order and using advanced interpolation methods. As a result, using the $4^{\text {th }}$ order HPF with the quadratic interpolation method shows the highest SNR improvement, whereas the $2^{\text {nd }}$ and $3^{\text {rd }}$ order HPFs show comparable results. In addition, the linear interpolation method leads to less area and lower power consumption compared to its quadratic counterpart, even though the quadratic interpolation method shows higher SNR improvements in many cases. However, the entire ECG baseline detection block can be replaced by a low-pass filter (LPF) that can track the baseline wander to a certain degree. Although this approach achieves the lowest power consumption and area, the accuracy of detecting the baseline wander (SNR improvement) is decreased drastically in comparison to other cases. This indicates simply using the LPF to extract the baseline wander from the corrupted ECG signal, instead of interpolating the down sampled moving average excluding the HF components will put more useful ECG features in the extracted baseline wander which will be subtracted from the corrupted ECG signal, thus resulting in a poor SNR improvement. Table 2 shows the performance of presented work with $4^{\text {th }}$ order HPF and quadratic interpolation compared to existing hardware-based baseline wander elimination schemes. It is clear that this work reduces the power consumption and chip area drastically while removing motion artifacts effectively.

Table 2. Performance comparison between the presented work and hardware implemented designs

\begin{tabular}{|c|c|c|c|}
\hline Ref & [6] & {$[12]$} & This work \\
\hline Technology & $0.13 \mu \mathrm{m}$ CMOS & $0.18 \mu \mathrm{m}$ CMOS & $0.18 \mu \mathrm{m}$ CMOS \\
\hline Supply & $1.2 \mathrm{~V}$ & $1.2 \mathrm{~V}$ & $1.8 \mathrm{~V}$ \\
\hline Power & $\begin{array}{c}46-73 \mu \mathrm{W} \\
+ \text { FPGA }\end{array}$ & $\begin{array}{c}374 \mu \mathrm{W} \\
+\mathrm{ARM} \text { Cortex- } \\
\text { M0 }\end{array}$ & $19.14 \mu W$ \\
\hline Area (IC only) & $2.35 \times 2.38 \mathrm{~mm}^{2 *}$ & $7 \times 7 \mathrm{~mm}^{2 * *}$ & $3.4 \times 4.6 \mathrm{~mm}^{2 * * *}$ \\
\hline $\begin{array}{l}\text { Motion } \\
\text { Artifact } \\
\text { Elimination }\end{array}$ & $\begin{array}{l}\text { can handle } 17 \times \\
\text { larger amplitude }\end{array}$ & $\begin{array}{c}\text { SAR } \\
\text { improvement } \\
8-10 \mathrm{~dB}\end{array}$ & $\begin{array}{c}\text { SNR } \\
\text { improvement } \\
9.96 \mathrm{~dB} \\
\end{array}$ \\
\hline
\end{tabular}

\section{CONCLUSION}

In this paper, a fully analog circuit which tracks and removes baseline wander from ECG signals corrupted with motion artifacts is presented. The baseline wander is extracted by down sampling the moving average obtained from the corrupted ECG waveform, and quadratically interpolating it, where the HPF is used to exclude the Rpeak samples from the moving average. The clean ECG waveform is generated by subtracting the detected baseline wander from the delayed version of the corrupted ECG waveform. Simulation results show the proposed circuit can effectively track and remove baseline wander from ECG waveforms corrupted with motion artifacts, where the $4^{\text {th }}$ order HPF along with quadratic interpolation structure has the best performance - SNR improvement around $10 \mathrm{~dB}$. The proposed circuit can be realized with low power and small area, thus can enable a very compact and energy efficient ambulatory ECG monitoring platform.

\section{REFERENCES}

[1] R. Kohno, H. Abe, and D. G. Benditt, "Ambulatory electrocardiogram monitoring devices for evaluating transient loss of consciousness or other related symptoms," Journal of arrhythmia, vol. 33, no. 6, pp. 583-589, Dec. 2017.

[2] S. Jain, V. Bajaj, and A. Kumar, "Riemann Liouvelle fractional integral based empirical mode decomposition for ECG denoising," IEEE journal of biomedical and health informatics, vol. 22, no. 4, pp. 1133-1139, Jul. 2018.

[3] M. Huang, D. Chen, and F. Xiong, "An Effective Adaptive Filter to Reduce Motion Artifacts from ECG Signals Using Accelerometer," in Proceedings of the 2019 9th International Conference on Biomedical Engineering and Technology, pp. 83-88, Mar. 2019.

[4] H. Sharma and K. Sharma, "Baseline wander removal of ECG signals using Hilbert vibration decomposition," Electronics Letters, vol. 51, no. 6, pp. 447-449, Mar. 2015.

[5] N. Van Helleputte, S. Kim, H. Kim, J. P. Kim, C. Van Hoof, and R. F. Yazicioglu, "A $160 \mu \mathrm{A}$ Biopotential Acquisition IC With Fully Integrated IA and Motion Artifact Suppression," IEEE transactions on biomedical circuits and systems, vol. 6, no. 6, pp. 552561, Dec. 2012.

[6] S. Hong, S. Lee, T. Roh, and H. Yoo, "A $46 \mu \mathrm{W}$ motion artifact reduction bio-signal sensor with ICA based adaptive DC level control for sleep monitoring system," IEEE CICC 2012, pp. 289-292, Sep. 2012.

[7] A. B. J. Teoh and L. Leng, "Editorial: Special Issue on Advanced Biometrics with Deep Learning," Applied Sciences, vol. 10, no. 13, pp. 4453_1-4, Jun. 2020.

[8] P. P. Roy, P. Kumar, and V. Chang, "A hybrid classifier combination for home automation using EEG signals," Neural Computing and Applications, pp. 1-13, Mar. 2020.

[9] P. Kumar, R. Saini, P. P. Roy, and D. P. Dogra, "A biosignal based framework to secure mobile devices," Journal of Network and Computer Applications, vol. 89, pp. 62-71, Jul. 2017.

[10] M. Nazari and K.-S. Lee, "Fully Analog Baseline Wander Elimination Circuit For Real-Time 
Ambulatory ECG Recording,” in 2019 IEEE 62nd International Midwest Symposium on Circuits and Systems (MWSCAS), pp. 33-36, Aug. 2019.

[11] P. D. Arini, S. Liberczuk, J. G. Mendieta, M. Santa María, and G. C. Bertrán, "Electrocardiogram delineation in a Wistar rat experimental model," Computational and mathematical methods in medicine, vol. 2018, Feb. 2018.

[12] N. Van Helleputte, M. Konijnenburg, J. Pettine, D.-W. Jee, H. Kim, A. Morgado, et al., "A $345 \mu \mathrm{W}$ multisensor biomedical SoC with bio-impedance, 3-channel ECG, motion artifact reduction, and integrated DSP," IEEE Journal of Solid-State Circuits, vol. 50, no. 1, pp. 230-244, Jan. 2015.

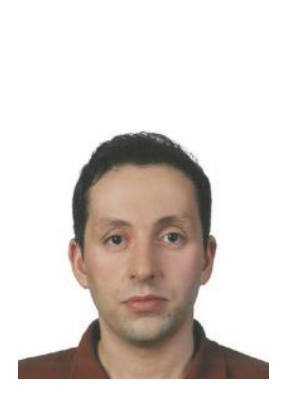

\section{Authors}

University of Akron, Akron, OH, USA

Masoud Nazari received his B.S. degree in 2011 from K. N. Toosi University of Technology and M.S. degree in 2014 from Shahid Beheshti University both in electrical engineering, Tehran, Iran. In 2017, he joined the Department of Electrical and Computer Engineering for pursuing his $\mathrm{PhD}$ degree at The

His research interests include low power analog and mixedsignal integrated circuits for biomedical applications.

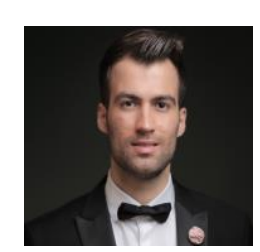

Alireza Bagheri Rajeoni received his B.S. degree in Electrical Engineering from the University of Guilan, Iran in 2015. He is currently pursuing his M.S. as a research assistant in Electronics Engineering at the University of Akron, $\mathrm{OH}$, USA.

His research interest includes low power analog circuits, bio-medical circuits, and machine learning circuits and systems.

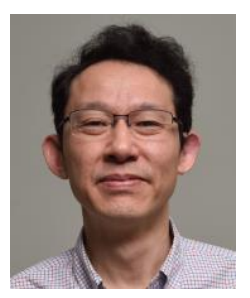

Kye-Shin Lee received the B.S. degree from Korea University, Seoul, Korea, in 1992, the M.S. degree from Texas A\&M University, College Station, TX, USA, in 2002, and the Ph.D. degree from the University of Texas at Dallas, Richardson, TX, USA, in 2005, all in electrical engineering.

$\mathrm{He}$ was with Texas Instruments Inc., Dallas, TX, USA, from 2005 to 2008. In 2009, he was an Assistant Professor with the Department of Electronics, Sun Moon University, Asan-si, Chungnam, Korea. He is currently an Associate Professor with the Department of Electrical and Computer Engineering, The University of Akron, Akron, OH, USA. He served as a guest editor for International Journal of Distributed Sensor Networks, and technical program committee for IEEE Midwest Symposium on Circuits and Systems (MWSCAS), and since 2018 he is serving as the region editor for Journal of Multimedia Information Systems. His research interests include analog integrated circuits and data converters for sensor applications, machine learning circuits and systems, and analog circuit design automation/methodology. 
Fully Analog ECG Baseline Wander Tracking and Removal Circuitry using HPF Based R-peak Detection and Quadratic Interpolation 